\title{
Simulation of Soft Tissue in 3D-Printed Models for Guided Bone Regeneration Education
}

\begin{abstract}
The use of custom-made 3D-printed models extracted from the Cone Beam Computed Tomography (CBCT) of the patient provides the dentist with the opportunity to rehearse a surgical procedure such as implant placement enhancing its tactile simulation in order to achieve a predictable and successful surgical outcome. Even the fact that these models have substantially improved the preparation and education in implant surgery, they lack the soft tissue layers necessary for simulating important procedure such as periosteal releasing incisions and flap primary closure. For a more realistic surgical simulation in implant education, the soft tissue layers of the mucoperiosteal flap have been incorporated into the existent 3D-printed model. The purpose of this report is to represent, describe, document and discuss the recreation of the soft tissue layers of the mucoperiosteal flap on a custom-made 3D-printed model extracted from the CBCT of the patient in a case used to simulate a guided bone regeneration (GBR) procedure.
\end{abstract}

\section{Introduction}

Implant dentistry is one of the most popular modalities in the dental field due to the high predictability and success rates of implants when compared to conventional treatment options to replace missing teeth in complete or partially edentulous patients [1-3].

In the last few years, new methods of diagnosis, treatment planning and execution techniques in dentistry have been developed or improved due to the advances in technology. Examples of this are the evolution of digital dental imaging from two-dimensional (2D) to three-dimensional (3D) images, the creation of digital dental impressions (intraoral scans), digital treatment planning (virtual software), digital guidance for surgery (computer-guided surgery) and digital 3D production (Computer-Aided Design/ComputerAided Manufacturing - CAD/CAM). Also, the recent advances in digital imaging such as Cone Beam Computed Tomography (CBCT) are improving the visualization, now with great details of the patient's anatomy in 3D when compared with standard radiographs, which are taken and seen in $2 \mathrm{D}[3]$.

As implant dentistry is becoming more widely practiced, effective continuous education is becoming very important, especially regarding the fact that implant dentistry as a main subject is usually not taught in undergraduate courses of dental schools, so clinicians who are going to practice it find the need for learning it afterwards either in postgraduate programs, advanced education training or weekend courses, among others. Mainly, this education is based on pre-clinical hands-on practice followed or not by clinical training. To design and perform a hands-on, several materials and methods can be used (typodonts, models from the mouth, animal jaws and human cadavers, among others). From these, the use of 3D-printed models

\section{Journal of Oral Biology}

\author{
Wangsrimongkol Buddhapoom, Reis Natacha, \\ Fernández-Guallart Ines, Yu Yung Cheng Paul* \\ Arthur Ashman Department of Periodontology and Implant \\ Dentistry, New York University, USA
}

*Address for Correspondence: Cheng Yu PY, Department of Periodontology and Implant Dentistry, New York University, Clinic 5W, 345 E 24th St, New York, NY 10010, New York, USA, Tel: +1-3472792958; E-mail: ycy233@nyu.edu

Submission: 16-April-2020

Accepted: 06-June-2020

Published: 09-June-2020

Copyright: $\odot 2020$ Wangsrimongkol B, et al. This is an open access article distributed under the Creative Commons Attribution License, which permits unrestricted use, distribution, and reproduction in any medium, provided the original work is properly cited.

extracted from patient's CBCT is becoming a well-accepted method of education because it allows to accurately simulate the real patient anatomy, especially in terms of hard tissues (teeth and bone), so procedures such as implant placement can be successfully practiced on them. However, the simulation of a complete implant surgical procedure with all its steps (incisions design, flap elevation, osteotomy, implant placement and suture, and in some cases, additional guided bone/tissue regeneration and/or sinus augmentation) is not possible in CBCT-based 3D-printed model hands-on due to a lack of soft tissues in these models, which management is of vital importance to achieve clinical success $[4,5]$.

Although the use of 3D-printed models is already established worldwide for surgical simulation in terms of education in implant dentistry, there is still shortage in publications regarding the best material for fabrication of soft tissues for these models. Park et al. (2017) reported the combination of vinyl tape, sponge tape and liquid tape to mimic the soft tissue in a 3D-printed model. However, there is a limitation in terms of the amount of periosteal releasing and consequential flap advancement that can be achieved in this option, which is only $1-2 \mathrm{~mm}$. In the new prototype presented in this article it was possible to gain $5-10 \mathrm{~mm}$ (similar to what can be achieved in a real patient), which is crucial for the achievement of tension-free primary closure [6].

The purpose of this report is to represent, describe, document and discuss the recreation of the soft tissue layers of the mucoperiosteal flap on a custom-made 3D-printed model extracted from the Cone Beam Computed Tomography (CBCT) of the patient in a case used to simulate a guided bone regeneration (GBR) procedure.

\section{Materials and Methods}

The steps for producing a 3D-printed model and simulating a surgical guided bone regeneration procedure on it are

1. Obtain a patient's CBCT scan using a CBCT imaging system (J. Morita, Kyoto, Japan), in which the field of view (FOV) of the image will be selected depending on the area of interest of the case.

2. Convert the Digital Imaging and Communications 
Citation: Wangsrimongkol B, Reis N, Fernández-Guallart I, Yu YCP. Simulation of Soft Tissue in 3D-Printed Models for Guided Bone Regeneration Education. J Oral Biol. 2020; 7(1): 4.

ISSN: 2377-987X

Table 1: Summary Table of Tissue Layers and Materials used for the fabrication of the 3D-Printed Model.

\begin{tabular}{|l|c|c|c|}
\hline Tissue Type & Tissue Layer & Material & Fabrication \\
\hline Hard Tissue & Bone (cancellous and cortical) & Grey Photopolymerized Resin & Stereolithography (STL) 3D-printed \\
\hline \multirow{2}{*}{ Soft Tissues } & Periosteum & White Liquid Tape & Added manually \\
\cline { 2 - 4 } & Connective Tissue and Epithelium & Orange Acrylic Paint & Painted manually \\
\hline
\end{tabular}

in Medicine (DICOM) file from the patient's CBCT into a Stereolithography (STL, OsiriX Lite, Geneva, Switzerland) format.

3. Process and print the STL file with a 3D- printer (Formlab Form 2 Printer, Somerville, Massachusetts, USA), using a grey methacrylic photoreactive liquid resin material that represents the hard tissue of the 3D-printed model.

4. Add acrylic paint to simulate soft tissue layers: periosteum is recreated with liquid tape and connective tissue and epithelium are reconstructed with orange acrylic paint, painted manually on the surface of the 3D-printed model (Tables 1).

5. A full-thickness midcrestal incision is made between the teeth adjacent to the defect. Two vertical incisions are made parallel or trapezoidal until the base of the flap, widening apically for easy coronal repositioning of the flap after augmentation with the graft material. After passing the imaginary mucogingival junction, the vertical incisions continue apically in a vertical or more convergent direction.

6. The liquid tape with orange acrylic paint is then elevated with a periosteum elevator.

7. Cortical perforation (decortication) is performed by \# 1 or \#2 round bur using high speed hand piece with copious irrigation.

8. Releasing incisions are made with a sharp 15C blade on the inner apical portion of the liquid tape and orange acrylic paint, creating a 2-3mm split-thickness dissection.

9. Tinfoil is used as a template, and in this case also as a mimic of the collagen membrane, fitted and trimmed to the ideal shape, and then put to the correct position. A single tack is positioned apically through the membrane into the photopolymerized resin, and the membrane is rotated 180 around the tack and adjusted to extend $2-3 \mathrm{~mm}$ beyond the augmented area. The graft material is placed and condensed to fill the defect.

10. Stabilization of the membrane and the underlying graft material is achieved by using horizontal mattress sutures extending from the apical portion of the liquid tape to the palatal aspect of the orange acrylic paint. Consequently, the liquid tape is then coronally advanced together with the orange acrylic paint, allowing tensionfree closure afterwards.

Finally, the complete adaption of the orange acrylic paint is achieved by multiple interrupted 4-0 chromic gut sutures.

In this case, the area of interest was the anterior maxilla, an aesthetic area where soft tissue management is usually necessary. Figure 1A shows the patient $\mathrm{CBCT}$, where it is possible to appreciate the selected area for the guided bone regeneration (GBR) surgical simulation. The DICOM file was processed and the STL file was printed obtaining the patient $3 \mathrm{D}$-model using a grey methacrylic photoreactive liquid resin material to mimic the hard tissue of the patient, as it is shown in Figure 1B. After the 3D- model was printed, the soft tissue layers needed for simulation of soft tissue management during GBR were fabricated using acrylic paint and white liquid tape. In this specific prototype, orange acrylic paint was used to reproduce the epithelium and connective tissue and liquid tape to reproduce the periosteum, as shown in Figure 1C (the white layer was the first one to be painted on the 3D model, and the orange layer was painted over it [Figure 2A]). In Table 1, the description of 3D model layers can be appreciated.

\section{Results}

In the present case report, the surgical procedure of GBR technique was performed successfully after the dentist did simulation and practised the surgery sequence in the enhanced 3D model.

Figure $1 \mathrm{~B}$ shows the hard tissues $3 \mathrm{D}$ model. Figure $1 \mathrm{C}$ shows the soft tissues 3D-printed model with the incisions design. Figure 2A shows flap elevation after midcrestal, mesial and distal incisions. Figure $2 \mathrm{~B}$ shows the periosteum releasing for flap advancement. Figure $2 \mathrm{C}$ shows the increased mobility of the flap to achieve tensionfree primary closure. Figure $3 \mathrm{~A}$ shows the occlusal view of the advancement of the flap in the 3D model, similar to the one achieved in the patient's mouth (Figure 3B).
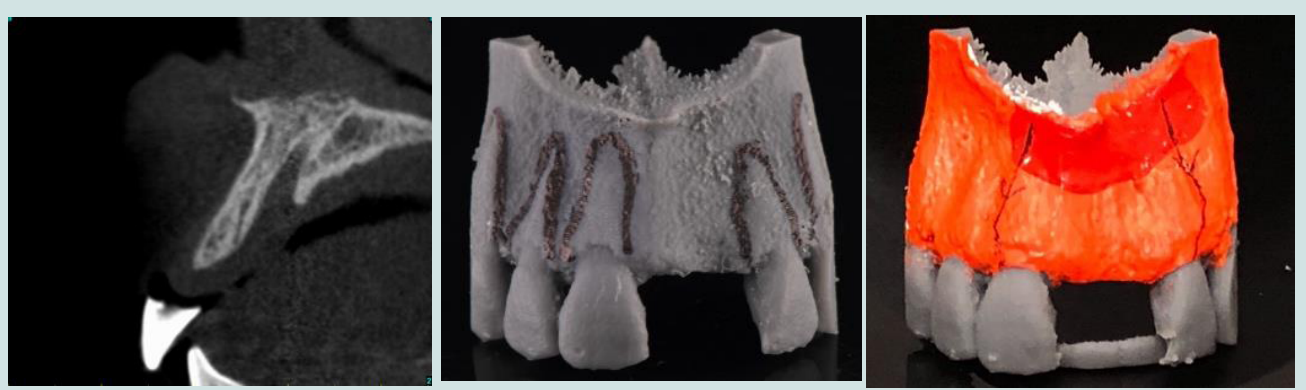

Figure 1: 1A- CBCT image of maxillary cross section; 1B - Current available 3D-printed model; 1C - Enhanced 3D-printed model. 

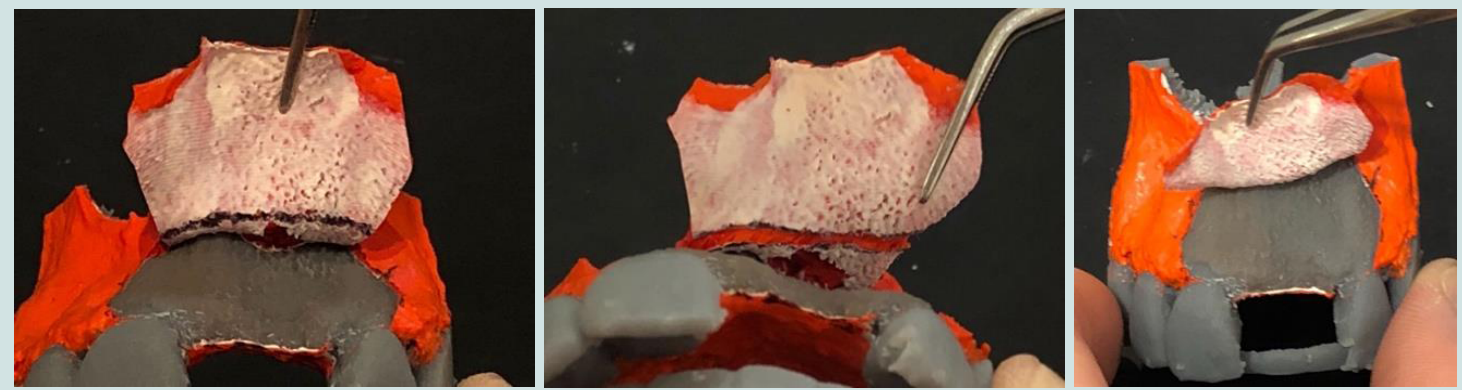

Figure 2: 2A - Simulation of a periosteal flap elevation on the 3D model; 2B - Simulation of a periosteal releasing incision on the 3D model; 2C - Increased mobility mobility of the flap after the releasing incision.

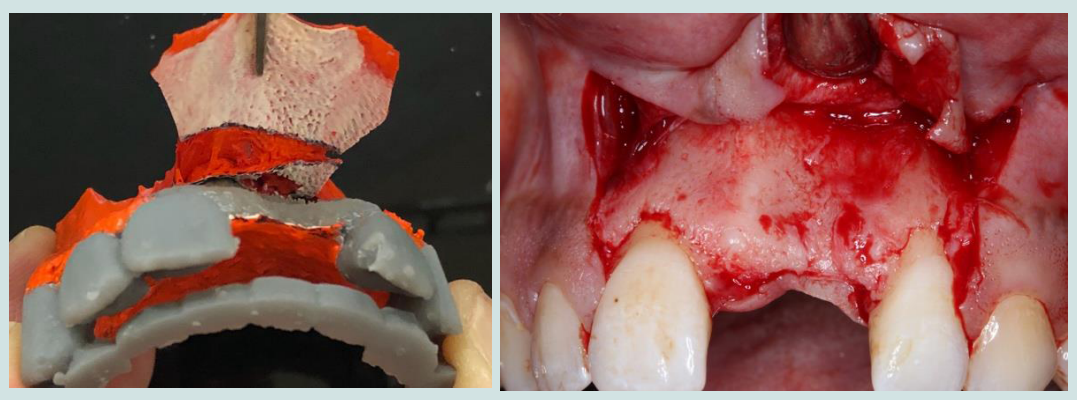

Figure 3: 3A - Simulation of flap advancement after a periosteal releasing incision on the 3D model; 3B - Periosteal incision in the patient's mouth as planned on the patient's 3D model.

\section{Discussion}

Nowadays, with the advances in technology, 3D imaging techniques are replacing $2 \mathrm{D}$ imaging techniques, especially in complex cases due to the need for better details of anatomy. CBCT scan is a $3 \mathrm{D}$ digital imaging technique that uses ionizing radiation (x-ray), although when compared with conventional CT scan it is less invasive in radiation exposure and has higher quality. The principle of the CBCT image is that higher density of the tissue equals to higher absorption of the radiation, so the performance in hard tissue imaging is higher than in soft tissue. CBCT is becoming more popular in implant dentistry due to the accurate visualization of hard tissues needed for implant treatment planning and moreover the possibility of virtual simulation of the implant position in relation to neighboring structures when introducing the $\mathrm{CBCT}$ data into a planning software. Magnetic Resonance Imaging (MRI) is a 3D digital imaging technique using non-ionizing radiation, which basic principle is the interaction between static magnetism and hydrogen atoms. Consequently, the higher amount of hydrogen atoms in the tissues, the higher density. Higher quantity of hydrogen atoms is found in tissues with higher quantity of water, so the performance in soft tissue imaging is higher than in hard tissues. In comparison with $\mathrm{CBCT}$, the imaging resolution of hard tissue is lesser, and it is also more expensive [7-9]. Both hard and soft tissue information are essential to produce a 3D-printed model. However, CBCT scan is preferable to MRI because without a highly precise hard tissue information from the real patient it is not possible to fabricate an actual 3D-printed model for surgery simulation.

Implant placement is a very straightforward surgical procedure in cases having enough bone width and height as well as healthy soft tissue. However, in more complex cases there is a need for reconstructing bony and soft tissue defects. TThere are several techniques for bone augmentation such as guided bone regeneration (GBR) with the use of membranes in combination to autogenous bone grafts, allografts and/or xenografts. To enhance its results bone promoting proteins (BPPs), or platelet rich plasma (PRP) can be used. Other techniques include inlay and onlay grafts, ridge splinting, and distraction osteogenesis among others. On the other hand, for soft tissue augmentation, there are also numerous techniques such as autogenous grafts (free gingival, connective tissue grafts), or the use of allogenic, xenogenic and synthetic materials. In all of these techniques, soft tissue management is needed in terms of incision making, flap elevation and suturing $[10,11]$.

Soft tissue management is important for a proper healing of the bone and soft tissue. Flap design with correct incisions is important for bony defect access, sustainability of the blood supply from the base of the flap, papillae preservation and free-tension primary closure allowance among others. After elevating a full-thickness mucoperiosteal flap, especially in cases of ridge augmentation, it is imperative to either stretch the elastic fibers present in the mucosa, or do a horizontal periosteal releasing incision in the apical part of the flap to increase the stretchability of the tissue in order to allow it to be advanced and cover all the surgical site, thus achieving the so-called tension-free primary closure. Primary closure should be tension-free in order to avoid pressure on the graft materials which can cause postoperative swelling, as well as to allow microvascular anastomosis for re-epithelization and fully wound closing, enhancing the re-establishment of the blood supply [12-14]. 
Citation: Wangsrimongkol B, Reis N, Fernández-Guallart I, Yu YCP. Simulation of Soft Tissue in 3D-Printed Models for Guided Bone Regeneration Education. J Oral Biol. 2020; 7(1): 4.

ISSN: $2377-987 X$

\section{Conclusion}

The use of 3D-printed models from CBCT images had several advantages in surgical simulation and training in the past in comparison to standard models and other hands-on modalities. CBCT scans are more favorable than MRI in terms of achieving hard tissues visualization in $3 \mathrm{D}$ and transferring it to a $3 \mathrm{D}$ model. However, the recent challenge is the addition of soft tissues into the $3 \mathrm{D}$ models. This new issue is required due to the importance of soft tissue management in many procedures in implant dentistry, and thus a proper 3D model prototype should simulate the soft tissue layers: periosteum, connective tissue and epithelium. This new 3D model prototype provides an excellent hands-on for educational purposes, as it allows the practitioner to study, simulate and rehearse several times the soft tissue management procedure until he/she the reaches the expected level of confidence, since multiple replicas of soft tissues layers can be fabricated. In the next coming years, with the integration of MRI into CBCT, the production of even more realistic soft tissue architecture for surgical simulation seems possible. However, despite the promising results of the proposed 3D enhanced model, further investigations at a higher level of evidence are necessary to validate this proposed $3 \mathrm{D}$ model.

\section{References}

1. Brånemark PI, Adell R, Breine U, Hansson BO, Lindström J, et al.(1969) Intra-osseous anchorage of dental prostheses: I. Experimental studies. Scand J PlastReconstr Surg 3: 81-100.

2. Buser D, Sennerby L, De Bruyn H (2017) Modern implant dentistry based on osseointegration: 50 years of progress, current trends and open questions. Periodontol 73: 7-21.

3. Froum SJ, Cho SC, Loomer PM, Georgantza A, Suziki T (2016) The use of $3 \mathrm{D}$ printing in dental implant education. 1-13.
4. Dawood A, Marti Marti B, Sauret-Jackson V, Darwood A (2015) 3D printing in dentistry. British Dental Journal 219: 521-529.

5. Werz SM, Zeichner SJ, Berg B-I, Zeilhofer H-F, Thieringer F (2018) 3D Printed Surgical Simulation Models as educational tool by maxillofacial surgeons. Eur J Dent Educ 22: 500-505.

6. Park N, Bhekare A, Yu YCP, Scolnick J, Loomer PM (2017) Fabrication of Soft Tissue Layers for 3D Printed Models in Dental Implant Education. J Oral Biol 4: 3.

7. Shah N, Bansal N, Logani A (2014) Recent advances in imaging technologies in dentistry. World J Radiol 6: 794-807.

8. Pauwels R, Araki K, Siewerdsen JH, Thongvigitmanee SS (2015) Technica aspects of dental CBCT: state of the art. Dentomaxillofac Radiol 44: 2014 2024.

9. Niraj LK, Patthi B, Singla A, Gupta R, Ali I, et al. (2016). MRI in Dentistry- A Future Towards Radiation Free Imaging - Systematic Review. J Clin and Diag Res 10: 14-19.

10. Esposito M, Grusovin MG, Felice P, Karatzopoulos G, Worthington HV (2009) Interventions for replacing missing teeth: horizontal and vertical bone augmentation techniques for dental implant treatment (Review). Cochrane Database of Syste Rev 4: CD003607.

11. Wolff J, Farré-Guasch E, Sándor GK, Gibbs S, Jager DJ, et al.(2016) Soft Tissue Augmentation Techniques and Materials Used in the Oral Cavity: An Overview. Implant Dent 25: 427-434

12. Froum SJ, Cho SC, Pariente L (2012) A surgical protocol for guided bone regeneration procedures using absorbable membranes to minimize and treat complications. 1-16.

13. Heller AL, Heller RL, Cook G, D'Orazio R, Rutkowski J (2000) Soft tissue management techniques for implant dentistry: A clinical guide. $\mathrm{J}$ of Oral Implantol 26: 91-103.

14. Plonka AB, Sheridan RA, Wang H-L (2017) Flap Designs for Flap Advancement during Implant Therapy: A Systematic Review. Implant Dent 26:145-152. 